\title{
LID and LETID evolution of PV modules during outdoor operation and indoor tests
}

\author{
Esther Fokuhl*, Daniel Philipp, Georg Mülhöfer, and Paul Gebhardt \\ Fraunhofer Institute for Solar Energy Systems, Heidenhofstr. 2, 79110 Freiburg, Germany
}

Received: 30 June 2021 / Received in final form: 18 October 2021 / Accepted: 2 November 2021

\begin{abstract}
Light Induced Degradation (LID) and Light and Elevated Temperature Induced Degradation (LETID) manifest with carrier injection due to light or forward bias and can lead to performance losses during the first months or years of operation in the field. We are investigating the effects of common LETID indoor test conditions and the module temperature under outdoor exposure on the evolution of BO LID and LETID over time. The investigations are based on experimental data from twelve structurally identical mono-crystalline and two multi-crystalline PERC PV modules, which underwent a detailed experiment including five different indoor test sequences and an outdoor test. Changes in the module performance are discussed based on the current knowledge on state transitions of the BO defect and LETID. Temporary recovery of the LETID defect was used to distinguish LETID from other degradation mechanisms. Our results confirm the importance of BO stabilization prior to LETID tests as it is included in the current IEC TS draft for LETID detection. We also show that too strong acceleration of the processes can lead to misinterpretation of LETID test results. Under dark storage conditions, destabilization of BO defects was found to already evolve at temperatures as low as $75^{\circ} \mathrm{C}$ and a likely alteration of subsequent LETID was observed. The performance changes under outdoor exposure can be explained with the same mechanisms as investigated under indoor experiments and reveal reversible seasonal recovery effects. Furthermore, the influence of different module operating temperatures on the evolution of both, BO LID and LETID is presented and evaluated.
\end{abstract}

Keywords: BO LID / LETID / PERC / PV modules

\section{Introduction}

Since the first publication on LETID in 2012 [1], progress has been achieved on the understanding and mitigation of this degradation effect. While the root cause is still under investigation, there is consensus about hydrogen playing an important role in the defect formation. Studies have shown possible ways to mitigate LETID, such as adapting temperature profiles in the firing process $[2,3]$ or using thinner wafers [4].

Current efforts in standardization are going to improve the comparability of LETID test results and enable investors to identify LETID sensitive PV modules [5].

It has been recently shown for PERC PV modules, that BO LID can not only have an impact on LETID test results but also lead to false fails in test sequences of IEC61215 [6,7].

In this study, we are comparing changes in module characteristics of ten mono-crystalline PERC PV modules under different indoor test conditions addressing LID and

\footnotetext{
* e-mail: esther.fokuhl@ise.fraunhofer.de
}

LETID. As FeB LID can be ruled out by dark storage before the measurements, the focus of this work is on $\mathrm{BO}$ LID and LETID and changes in the module performance are discussed based on the current knowledge on possible state transitions of these effects.

We are also presenting and discussing performance changes of two PV modules of the same type as subjected to the indoor experiments and two strongly LETID sensitive multi-crystalline PERC PV modules under realistic outdoor exposure.

\subsection{State transitions of the $B O$ defect}

Possible state transitions of the BO defect can be illustrated by applying a three-state model [8]. As sketched in Figure 1, BO defects can be present in the states A, B or C. In the annealed state A, boron and oxygen are present as less recombination active, dissociated constituents. Degradation $\left(\overrightarrow{A B} \overrightarrow{B O}_{B O}\right)$ leads to the formation of the $\mathrm{BO}$ defect (state $\mathrm{B}$ ), which is highly recombination active. Passivation of the defect leads to a less recombination active, regenerated state $\mathrm{C}$. 


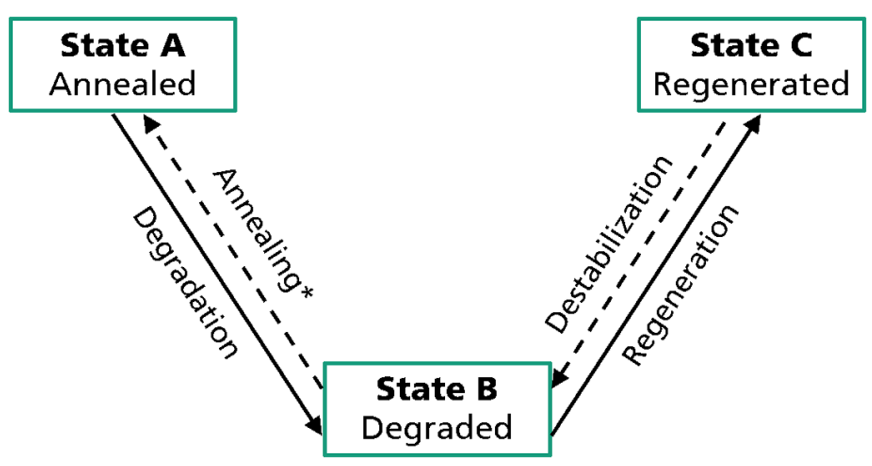

Fig. 1. Three-state model for the BO defect adapted from [16]. *When discussing similar state transitions of the LETID defect, we are using the term "Temporary Recovery" for transitions from B to A.

We consider the following state transitions to be relevant for our investigations:

Degradation $\left(\overrightarrow{A B} B_{B O}\right)$ is caused by excess carrier injection and can therefore be induced by irradiance with photon energy above bandgap or forward bias. The process is already activated at comparably low injection levels: At an irradiance above $10 \mathrm{~W} / \mathrm{m}^{2}$ the degradation was found to be almost independent from light intensity [9], though at high intensity light, an acceleration by increasing the irradiance level is possible [10]. The degradation rate is temperature dependent with Arrhenius behavior $[11,12]$.

Regeneration of the $\mathrm{BO}$ defect $\left(\overrightarrow{B C}_{B O}\right)$ has been reported to evolve at temperatures of $65^{\circ} \mathrm{C}$ or higher and simultaneous carrier injection [8]. The regeneration rate is highly temperature dependent with Arrhenius behavior $[8,13]$ and increases proportionally with the injection level [14]. It is assumed, that the underlying process is passivation of the defects by hydrogen [15].

Destabilization $\left(\overrightarrow{C B_{B O}}\right)$ and Annealing $(\overrightarrow{B A} B O)$ are solely thermally activated [16]. It has been shown for PERC PV modules in damp heat tests, that transitions from the regenerated to the annealed state can already evolve at $85^{\circ} \mathrm{C}[6,7]$.

Boron doped Cz-Si PV modules on the market usually contain a mix of $\mathrm{BO}$ defects in all states (A, B and C). Due to common regeneration procedures in the factory, a large share of defects is usually in the regenerated state C.

\subsection{State transitions of the LETID defect}

Like the BO defect, the LETID defect can be present in an annealed, degraded or regenerated state. Degradation $\left(\overrightarrow{A B}_{\text {LETID }}\right)$ is carrier induced and can therefore like $\mathrm{BO}$ LID be caused by either irradiation or forward bias. Compared to BO LID, LETID happens on significantly longer timescales [17]. Within usual experimental timeframes, it has been mainly observed at temperatures above approximately $50^{\circ} \mathrm{C}[1]$.

The degradation rate has been shown to have an Arrhenius-like temperature dependency and to be almost linearly dependent on the excess charge carrier density $[18,19]$.
It has been shown for temperatures $\geq 130{ }^{\circ} \mathrm{C}$, that LETID degradation can also be thermally activated without external carrier injection [20,21].

Regeneration of the LETID defect $\left(\overrightarrow{B C}_{L E T I D}\right)$ is accelerated by the same factors as degradation. The regeneration process happens more slowly compared to degradation and is like the degradation rate temperature and injection level dependent [17,18]. Like LETID degradation, also regeneration has been reported to happen under high temperature without carrier injection [20,21].

Carrier injection at room temperature has been shown to cause transitions from the degraded state $\mathrm{B}$ to the annealed state A $\left(\overrightarrow{B A}_{\text {LETID }}\right)$ temperature $[22,23]$. The kinetics of this temporary recovery have been shown to be highly injection level dependent [23].

Dark annealing has been shown to not only cause degradation and regeneration but also potentially alter the rate and severity of subsequent LETID $[20,24,25]$.

\subsection{BO LID and LETID in standardization}

Fast proceeding LID effects like BO LID are addressed in the light stabilization procedure of IEC61215-2 MQT 19 [26]. The module temperature of $(50 \pm 10){ }^{\circ} \mathrm{C}$ and the timescales of the stabilization procedure are however not in a range which would allow reliable conclusions on LETID susceptibility.

For LETID, a testing standard is of great importance, as the test conditions can have a high impact on the results [27].

For IEC standardization, a technical specification (TS) has been developed [5] based on previous work such as a LETID round robin [28] and an internal standard by TÜV Rheinland [29]. In the IEC TS draft the module temperature is controlled at $75^{\circ} \mathrm{C}$ while a current of $2 \times\left(I_{S C^{-}} I_{M P P}\right)$ is injected. Before the LETID stress test, BO preconditioning is performed as CID at $T \leq 30^{\circ} \mathrm{C}$ and $I_{S C}$ to separate LETID from BO LID.

Besides causing losses under field conditions, LID and LETID can also influence test results from module quality tests in IEC 61215. It has been shown that PERC solar cells with $\mathrm{BO}$ defects in the regenerated state can become sensitive for BO LID after damp heat testing due to destabilization at $85^{\circ} \mathrm{C}[6]$. A possible influence of $\mathrm{BO}$ LID and LETID on IEC test results has been recently investigated based on simulation results [7]. As a result of the investigations, an optional BO LID stabilization step has been added to IEC 61215:2021-2 (MQT19.3) after PID or Damp Heat [26]. The stabilization is performed with CID at $85^{\circ} \mathrm{C}$ and a current of $I_{S C}$.

\section{Experimental approach}

\subsection{Indoor test conditions}

Ten monocrystalline PERC PV modules of the same manufacturer and type have been tested under five different indoor test conditions known to cause LID and LETID (see Fig. 2). Two more PV modules of the same type have been installed in an outdoor test in May 2020. 


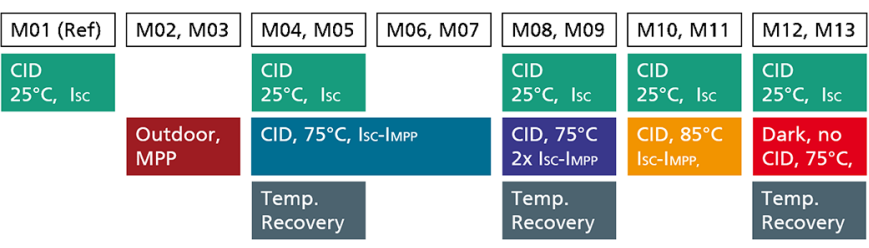

Fig. 2. Overview over the experiment.

One module of the same batch was used as reference module and characterized along with the modules from the experiments.

Test conditions were chosen according to relevant standards and draft standards (modules M04-M09), to investigate the influence of LETID testing at higher temperature on the test result (M10, M11) and to investigate effects of dark storage at increased temperature (M12, M13).

All indoor tests have been performed under dark carrier injection conditions ("Carrier Induced Degradation", CID).

During storage times, the modules were usually aligned in parallel on a trolley while the sunny side of the front module and the rear side of the rear module were covered with cardboard. This way, a possible influence of room light was reduced but not completely excluded.

The reference module (M01) and eight modules of the subsequent indoor tests (M04, M05, M08-M13) underwent at least $96 \mathrm{~h}$ of CID at $(25+9 /-2){ }^{\circ} \mathrm{C}$ (after steady state was reached in the climatic chamber) and a current injection of $I_{S C}$. The test was performed until no significant performance change was observed between the last two characterization measurements.

Four modules with (M04, M05) and without (M06, M07) prior BO LID preconditioning were subjected to CID at $(75 \pm 3)^{\circ} \mathrm{C}$ with an injection current of $I_{S C} I_{M P P}$ ("MPP mode"). These test conditions were originally suggested by Hanwha Q CELLS [17] and later tested in a round robin [28]. In a previous comparison of test methods [27], we had used these test conditions as reference conditions.

Two PV modules (M10, M11) have been tested at an increased temperature of $(85+1 /-4){ }^{\circ} \mathrm{C}$ in MPP mode after previous BO LID preconditioning. After our previous investigations [27] we had evaluated these test conditions as a possible accelerated LETID test.

The modules M08, M09 underwent CID at $(75 \pm 3){ }^{\circ} \mathrm{C}$ with an increased current of $2 \times\left(I_{S C}-I_{M P P}\right)$ after the BO LID preconditioning test. These are the test conditions used in the new IEC TS draft for LETID detection (see Sect. 1.3).

To investigate possible dark storage effects at elevated temperature, two PV modules (M12, M13) underwent dark storage at $(75+1 /-3)^{\circ} \mathrm{C}$ after $\mathrm{BO}$ LID stabilization. Except for in-situ dark IV measurements, which have been performed on module M13 every thirty minutes during most of the test intervals, no external carriers were injected during dark storage.

To trigger temporary recovery of the LETID defect $\left(\overrightarrow{B A}_{\text {LETID }}\right)$, CID at low temperatures was performed on six PV modules (M04-M05, M08-M09, M12-M13) after

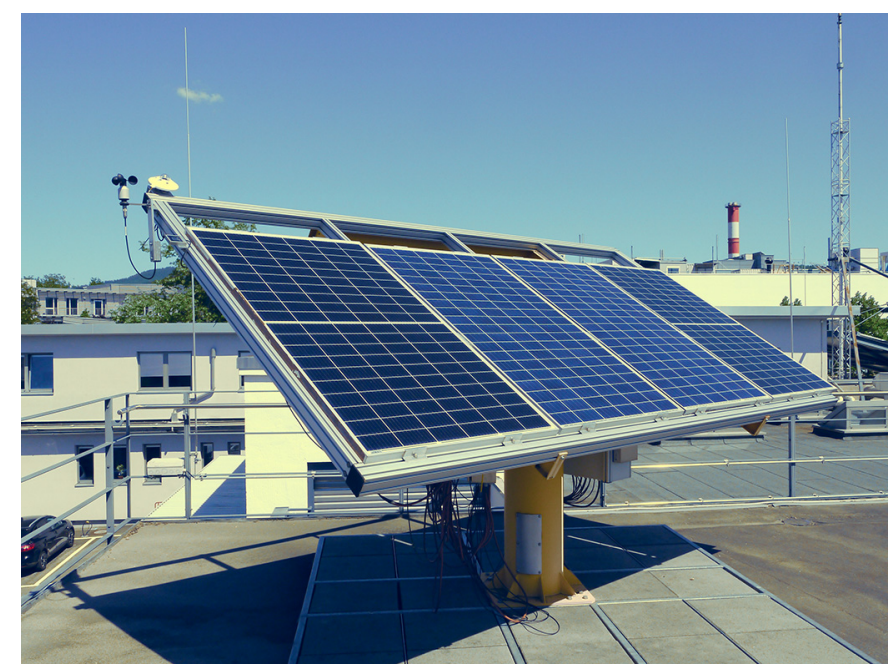

Fig. 3. PV modules on a two-axis tracker, which is used for the LETID outdoor test.

LETID testing (CID at $75^{\circ} \mathrm{C}$ and MPP mode or increased injection) and dark storage $\left(75^{\circ} \mathrm{C}\right)$. The main purpose of the experiment was to separate degradation due to $\mathrm{BO}$ LID and LETID after the previous experiments as it has been shown by others [30]. During the temporary recovery experiment, the module temperature was controlled in a range significantly lower than the temperatures relevant for degradation of the LETID defect and a current of $I_{S C^{-}} I_{M P P}$ ("slow temporary recovery test") or $I_{S C}$ ("fast temporary recovery test") was injected.

\subsection{Outdoor test}

Two PV modules (M02, M03) from the same type and manufacturer as the modules used for the indoor LID and LETID experiments have been installed on a two-axis tracker (see Fig. 3) at an outdoor test site in Freiburg, Germany in May 2020. On the tracker, also two LETIDsensitive multi-crystalline PERC PV modules have been monitored since April 2019 [31]. From each module type, one module has been thermally insulated on the rear side to show the effect of different operating temperatures. All modules are tracked in their MPP by electronic loads. Performance changes are monitored by regularly power measurements at STC as well as electroluminescence measurements.

During exposure time, module temperatures and in-plane-irradiance are monitored.

\subsection{Characterization methods}

Before and after each indoor test interval and each outdoor exposure interval, performance measurements at STC were performed with overall measurement uncertainties of $1.8 \%$ and a long-term reproducibility below $\pm 0.5 \%$. One reference module of each type was measured along with the modules. In case of the monocrystalline modules, the 


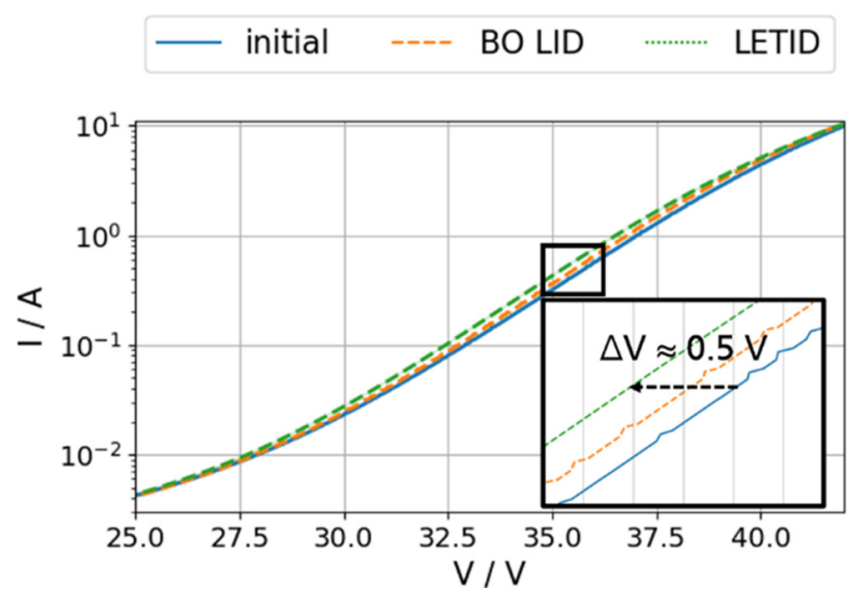

Fig. 4. Temperature corrected dark IV curves of module M04 before the experiment, after BO preconditioning and after LETID testing, shown for $V \geq 25 \mathrm{~V}$.

reference module was stabilized by BO LID preconditioning at the beginning of the experiments. Between the measurements, the reference module was stored along with the test modules (see Sect. 2.1). At each characterization step, electroluminescence images were taken while the characterized module was operated in $1.0 \times I_{S C}$ and $0.1 \times I_{S C}$.

Dark IV measurements were performed in-situ during the most indoor test intervals on at least one module of each test condition. During the measurement, the voltage step size was set to $0.1 \mathrm{~V}$ and the current was limited to $10 \mathrm{~A}$. In most of the tests, dark IV measurements were triggered every 30 minutes and a typical measurement time for a forward and reverse swipe was $42 \mathrm{~s}$. This information is relevant to evaluate possible influence due to carrier injection during dark IV measurements. In addition to the in-situ measurements, dark IV curves were also measured between the test and exposure intervals at a temperature of $(22 \pm 4)^{\circ} \mathrm{C}$.

\section{Indoor test results}

\subsection{Dark IV characteristic and dark voltage}

According to the current standards and draft standards, it is recommended to monitor and analyze the in-situ dark voltage during LETID tests in order to detect, when the maximum degradation has been reached $[5,29]$. The link between degradation during the test and the dark voltage at constant current level can be illustrated on the basis of the dark IV characteristics.

In Figure 4, dark IV curves of one module before the experiment, after $\mathrm{BO}$ preconditioning and after LETID testing are shown in the voltage range above $25 \mathrm{~V}$. The voltage was temperature corrected using current-dependent temperature coefficients for the voltage, which were derived from measurements on one of the PV modules.

Taking the two-diode-model as basis [32], the strongest changes in the dark IV curve can be viewed in the voltage range in which the parameters of the first diode dominate

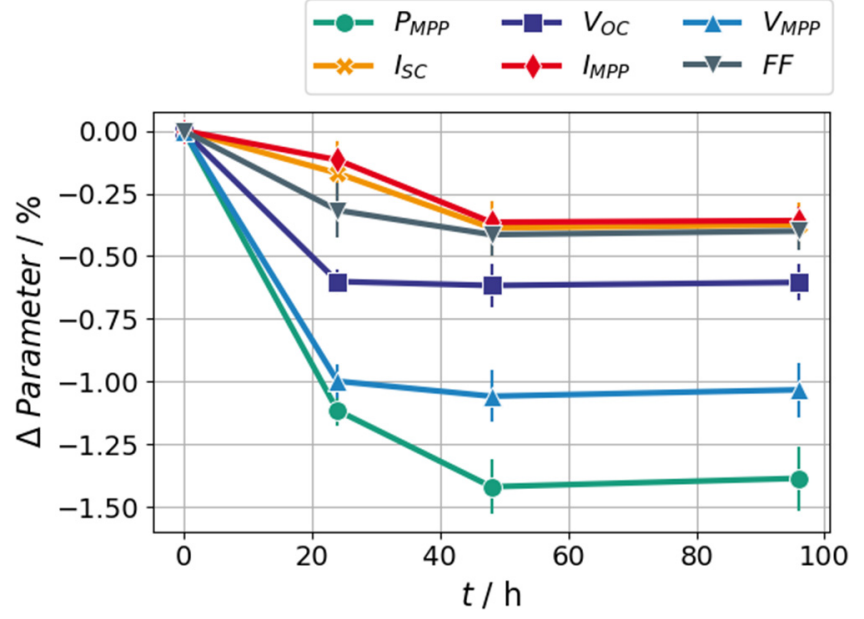

Fig. 5. Relative changes of the STC performance parameters of modules M04, M05 and M08 - M13 due to BO LID preconditioning at $25^{\circ} \mathrm{C}$ and $I_{S C}$. Average values over all modules; Error bars: standard deviation.

the dark IV characteristics. This corresponds well with the theory on BO LID and LETID, as both defects increase the recombination in the bulk [17].

The MPP voltage at STC $\left(V_{M P P, \text { initial }} \approx 34.3 \mathrm{~V}\right)$ is in the voltage range most sensitive for degradation due to LID and LETID. During CID at typical LETID test conditions, the module is operated at constant current in a point of operation close to the MPP voltage. The evolution of the dark voltage can therefore be used as an indicator for the progress of degradation.

In the example in Figure 4, a voltage decrease of approximately $0.5 \mathrm{~V}$ can be observed after BO LID preconditioning and LETID at a constant current of $I_{S C}-I_{M P P}$. This value corresponds to a relative voltage decrease of $-1.4 \%$, while at STC, $V_{M P P}$ losses of $-1.9 \%$ were measured.

For the interpretation of the test results discussed in Section 3.2, the in-situ measured dark voltage and dark IV characteristic are used as additional information on the proceeding of performance changes during the experiments.

\subsection{Performance changes}

\subsubsection{BO LID preconditioning}

Figure 5 shows the changes in the IV curve parameters at STC measured between BO LID preconditioning intervals at $25^{\circ} \mathrm{C}$ and $I_{S C}$ of eight modules from the experiment. After $48 \mathrm{~h}$ of CID, a stabilized state was reached. The degradation process due to BO LID has therefore most likely stabilized during the time interval between $24 \mathrm{~h}$ and $48 \mathrm{~h}$.

After $96 \mathrm{~h}$, the mean maximum power degradation amounts $-1.4 \%$ with a high-low span from $-1.1 \%$ to $-1.5 \%$. The power losses can be assigned to losses in $V_{O C}$ $(\approx-0.6 \%), F F(\approx-0.4 \%)$ and $I_{S C}(\approx-0.4 \%)$. As the voltage in MPP is mainly influenced by both, $V_{O C}$ and $F F$, 


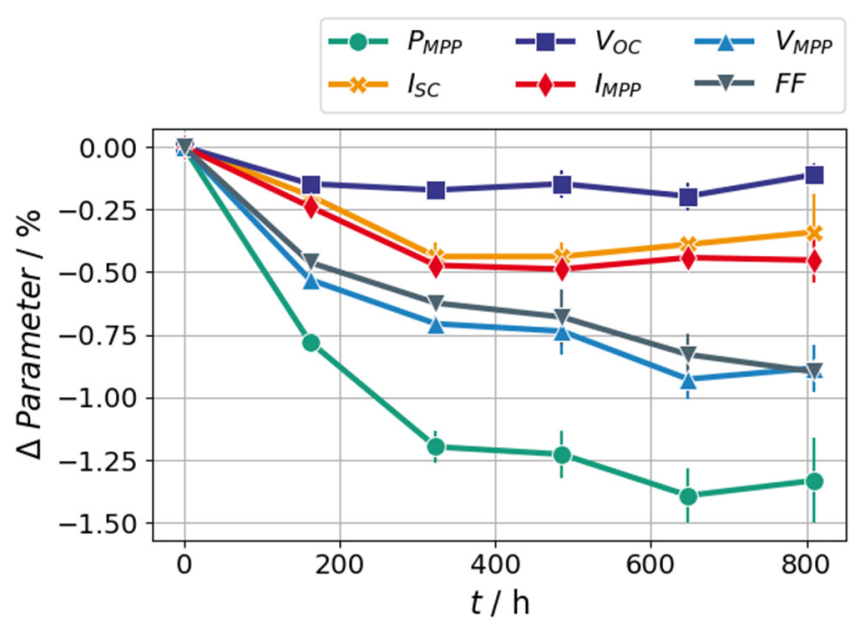

Fig. 6. Relative changes of the STC performance parameters of modules M04 and M05 during LETID testing at $75^{\circ} \mathrm{C}$ and MPP mode after BO LID stabilization. Average values over two modules; Error bars: min / max values of the two modules.

this value appears to behave comparably sensitive to the degradation of the tested modules. This finding matches well with the sensitivity of the dark IV characteristic at MPP voltage.

The error bars show the computed standard deviations for the eight modules at each intermediate measurement and indicate a high consistency between the tested modules. The electroluminescence images, however, show significant differences between the cells in each module (not shown). The comparably low module-to-module differences might be explained by a balanced distribution of cells with different amount of BO LID.

\subsubsection{LETID test at $75^{\circ} \mathrm{C}$ and MPP mode}

In the following LETID test at $75^{\circ} \mathrm{C}$ two modules (M04, M05) show additional power losses between $-1.2 \%$ and $-1.5 \%$ with FF losses being the highest contributor (see Fig. 6). Again, $V_{M P P}$ is the parameter most sensitive to the observed degradation. After the last test interval, M04 shows a slightly positive power change indicating that the maximum degradation has been reached during the last two test intervals.

In the following, the influence of BO LID preconditioning on LETID test results is demonstrated based on the performance changes of the modules M04, M05, M06 and M07 during LETID testing at $75^{\circ} \mathrm{C}$ and MPP Mode with and without prior BO CID.

Figure 7 shows the progress of STC power loss during LETID testing of the two pre-stabilized PV modules compared to two modules without BO LID preconditioning (M06, M07). The performance change of the prestabilized modules is referred to the value after BO LID stabilization while the changes of the non-stabilized PV modules are referred to the initial power measurement. Due to a storage time of 7 weeks, some BO LID already occurred on the non-stabilized modules before the start of the test. During the test, the power loss evolves to a maximum value between $-2.7 \%$ and $-2.9 \%$. The

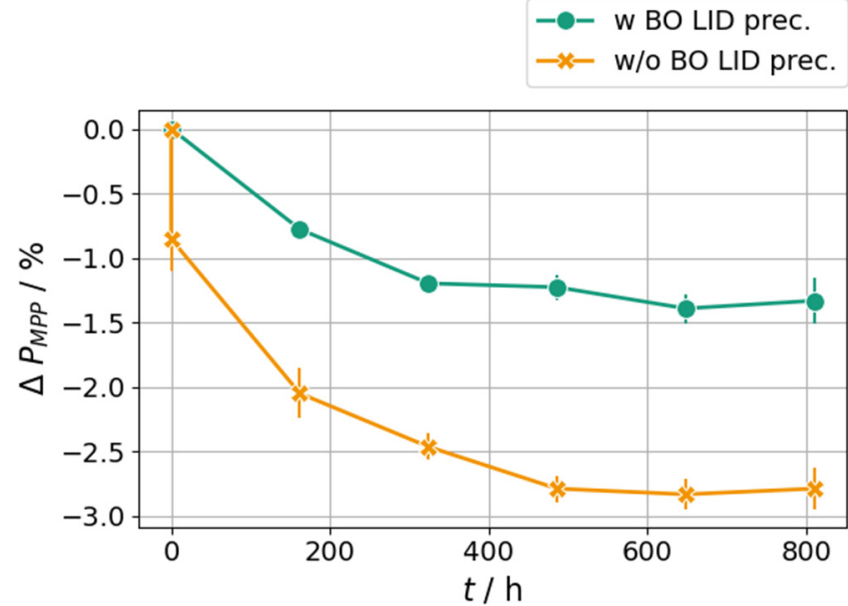

Fig. 7. Relative changes of $P_{M P P}$ at STC of modules during LETID testing at $75^{\circ} \mathrm{C}$ and MPP mode with (M04, M05) and without (M06, M07) prior BO LID stabilization. Average values over two modules; Error bars: min / max values of two modules.

difference between the mean values of the maximum degradation of the pre-stabilized and the non-stabilized modules amounts $1.5 \%$, which is in the range of the maximum degradation after BO LID preconditioning.

This demonstrates how degradation due to BO LID can influence the interpretation of LETID test results. Without knowing the share of BO LID, the LETID sensitivity of PV modules can be overestimated. In the IEC TS draft, this is taken into account by including a BO preconditioning step prior to the LETID stress test (see Sect. 1.3).

\subsubsection{LETID test at $85^{\circ} \mathrm{C}$ and MPP mode}

At an increased temperature of $85^{\circ} \mathrm{C}$, an acceleration of the processes was expected. Therefore, smaller test intervals between intermediate measurements were chosen compared to the test at $75^{\circ} \mathrm{C}$.

During the first $262 \mathrm{~h}, P_{M P P}$ drops by $-1.5 \%$, which is comparable to the maximum degradation due to LETID observed at $75^{\circ} \mathrm{C}$ (see Fig. 8). After the last test interval, further performance losses led to an overall power change of $-1.9 \%$ during $360 \mathrm{~h}$ of testing time. The higher temperature therefore did not only influence the degradation speed but also the amount of degradation. One possible explanation could be the activation of additional degradation types beyond LETID as suggested in literature [20], though, we did not verify this hypothesis by further experiments on M10 and M11 in scope of this work.

\subsubsection{LETID test at $75^{\circ} \mathrm{C}$ and $2 \times\left(I_{S C}-I_{M P P}\right)$}

At $75^{\circ} \mathrm{C}$ and $2 \times\left(I_{S C}-I_{M P P}\right)$, a maximum power loss between $-0.3 \%$ and $-0.5 \%$ was measured after $96 \mathrm{~h}$ (see Fig. 9). After a storage time of four months before the second test interval slightly lower performance values were measured which led to the mean value of $-0.5 \%$ relative $P_{M P P}$ change included in Figure 9 . This is the lowest value measured during the test. According to the 


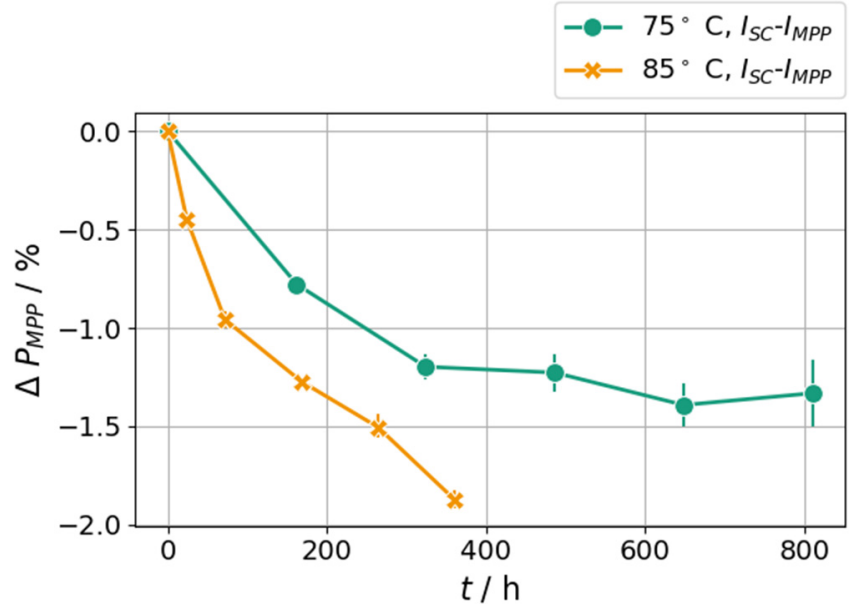

Fig. 8. Relative changes of $P_{M P P}$ at STC of PV modules during LETID testing at $75^{\circ} \mathrm{C}(\mathrm{M} 04, \mathrm{M} 05)$ and at $85^{\circ} \mathrm{C}(\mathrm{M} 10, \mathrm{M} 11)$ and MPP mode. Average values over two modules; Error bars: min/ max values of two modules.

evolution of the monitored dark voltage of module M09 (see Appendix A in Supplementary Material), the maximum power loss must have been reached close to the first performance measurement at STC. Thus, the increased current led to less power degradation than in MPP mode.

This difference in the magnitude of performance loss was stronger than expected, since other results known from literature (e.g., [17,33]) showed a significantly lower influence of the injection level on the amount of LETID. Therefore, further investigations have been performed on M08 and M09.

Assuming an injection level dependency of LETID degradation, LETID regeneration and BO LID regeneration, the lower performance loss might be explained by acceleration of either BO or LETID regeneration. In order to attribute the remaining power loss to either BO LID or LETID, a temporary recovery test was performed later on the two modules tested at $75^{\circ} \mathrm{C}$ and $2 \times\left(I_{S C}-I_{M P P}\right)$ (M08, M09) and the two modules tested at $75^{\circ} \mathrm{C}$ in MPP mode after BO LID preconditioning (M04, M05) (see Sect. 3.2.7). Based on the insights from the temporary recovery test, the result from Figure 9 is going to be further discussed.

\subsubsection{Dark storage at $75{ }^{\circ} \mathrm{C}$}

In the dark storage experiment at $75^{\circ} \mathrm{C}$, we observed a strong influence by carrier injection due to in situ dark IV measurements as well as laboratory storage times and intermediate measurements. These influences can likely be explained by BO LID, as discussed below.

The relative power change at STC measured after dark storage intervals of two modules with (M13) and without dark IV monitoring (M12) is shown in Figure 10. Before the experiment, both modules had undergone BO LID preconditioning (see Sect. 3.2.1). After each interval with dark IV monitoring (all intervals, except interval 2), module M13 shows a performance decrease. After $1134 \mathrm{~h}$ of dark storage, the $P_{M P P}$ change of M13 dark IV monitoring

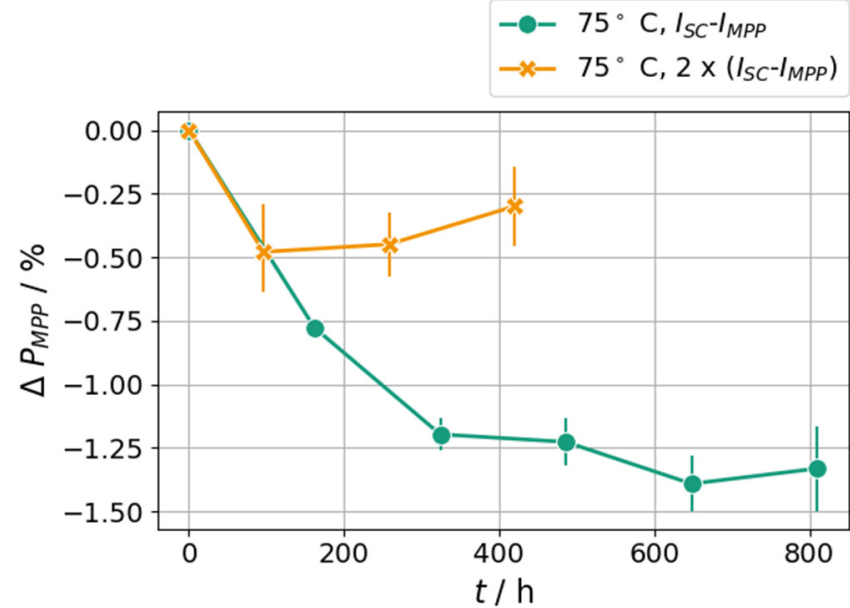

Fig. 9. Relative changes of $P_{M P P}$ at STC of PV modules during LETID testing at $75^{\circ} \mathrm{C}$ and MPP mode (M04, M05) and at $75^{\circ} \mathrm{C}$ and $2 \times\left(I_{S C}-I_{M P P}\right)(\mathrm{M} 08, \mathrm{M} 09)$. Average values over two modules; Error bars: min / max values of two modules.

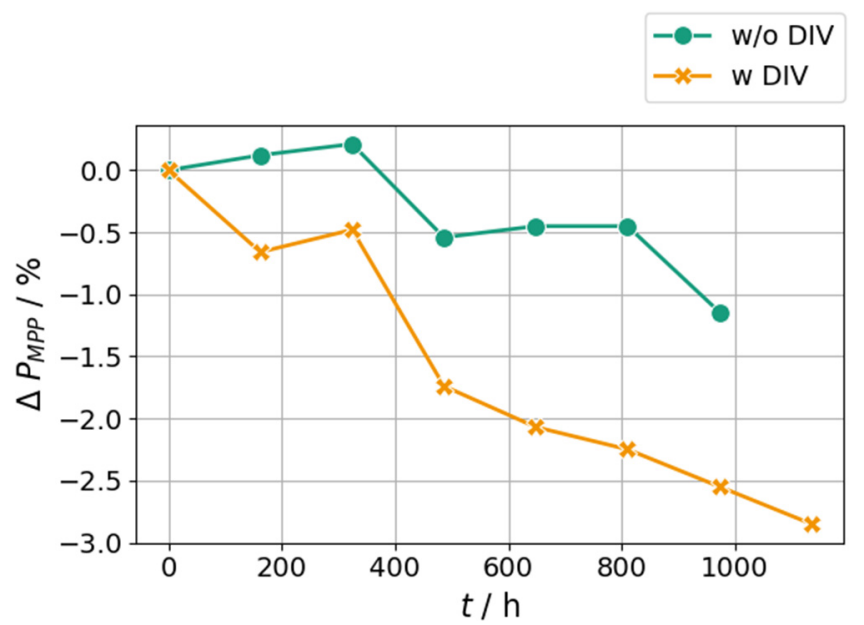

Fig. 10. Relative changes of $P_{M P P}$ at STC of two modules during dark storage at $75^{\circ} \mathrm{C}$ with (M13) and without DIV monitoring (M12).

amounts to $-2.8 \%$, which is approximately double the power loss observed under the LETID test conditions $75^{\circ} \mathrm{C}$ and MPP mode. The continuous voltage drop in the dark IV curve at $I_{S C}-I_{M P P}$ (see Appendix A in Supplementary Material) indicates, that a major proportion of this degradation has evolved during the test intervals and not during storage or intermediate measurements.

After test interval $3(t=486 \mathrm{~h})$ and prior storage time of 12 weeks, both modules show significant power changes compared to the previous measurement in the range of $-0.8 \%$ (module M12, without dark IV monitoring) and $-1.3 \%$ (module M13, with dark IV monitoring). The other test intervals at $t \leq 810 \mathrm{~h}$ did not lead to noticeable degradation of module M12 (without dark IV monitoring). After the last test intervals, however a $P_{M P P}$ change of $-0.7 \%$ was measured. 
As addressed in Section 1.1 dark storage at elevated temperatures can cause transitions of the boron oxygen defect from the regenerated to the annealed state. If this defect destabilization and annealing is already triggered at temperatures in the range of $75^{\circ} \mathrm{C}$, subsequent carrier injection is likely to cause degradation due to BO LID. This is a possible explanation for the performance loss of module M12 after interval 3 and interval 6.

The continuous power loss of module M13, on which dark IV monitoring was performed, might be explained by the same phenomenon: During each dark IV measurement, carriers were injected for an average time of $25 \mathrm{~s}$ in the first test interval and for $42 \mathrm{~s}$ in all other test intervals. During $1134 \mathrm{~h}$ of dark storage at $75^{\circ} \mathrm{C}$, the cumulative time with carrier injection due to dark IV amounts to $19 \mathrm{~h}$. During the investigations on the temperature dependence of the dark IV characteristic, carriers were injected for an additional time of $4.4 \mathrm{~h}$. Therefore, after each 30 minutes of dark storage at $75^{\circ} \mathrm{C}$, which might have triggered $\mathrm{BO}$ destabilization and annealing $\left(\overrightarrow{C B}_{B O}, \overrightarrow{B A} A_{B O}\right)$, the carrier injection during the measurement might have caused some $\mathrm{BO}$ defects to transit from the annealed state to the degraded state $(\overrightarrow{A B} B O)$.

The carrier injection during dark IV measurements might also have caused degradation due to LETID. However, as the overall performance loss is higher than observed for the modules under LETID test conditions, LETID can probably only explain part of the degradation. Considering the long timescales for LETID, it is more likely, that a great portion of the degradation is caused by BO LID after destabilization.

In order to investigate which degradation mechanisms are responsible for the performance losses, module M13 has been subjected to a temporary recovery test (see Sect. 3.2.7).

\subsubsection{CID after dark storage}

After six weeks of dark storage at $75^{\circ} \mathrm{C}$, module M12, which had been tested without in situ dark IV monitoring, was accidently subjected to $162 \mathrm{~h}$ of CID at $75^{\circ} \mathrm{C}$ in MPP mode during the last dark storage test interval. This led to an additional power loss of $-2.9 \%$, which is approximately double the maximum performance loss of the pre-stabilized modules without prior high temperature dark storage under these test conditions.

We consider two possible explanations for this comparably high performance change: Assuming, that some BO defects, which were destabilized and annealed during high temperature dark storage, were still in the annealed state before CID, these defects would have been changed to the degraded state during CID at $75{ }^{\circ} \mathrm{C}$ $(\overrightarrow{A B} B O)$. The combination of LETID in a range, as observed at CID without prior high temperature dark storage, and further BO LID could have led to the overall performance loss of $-2.9 \%$ during the test interval.

Another explanation could be an influence of dark storage at $75^{\circ} \mathrm{C}$ on the kinetics and extent of subsequent LETID. As it has been shown in literature, both can be highly influenced by dark annealing $[20,24,25]$. Though the dark anneal before LETID in these studies has been

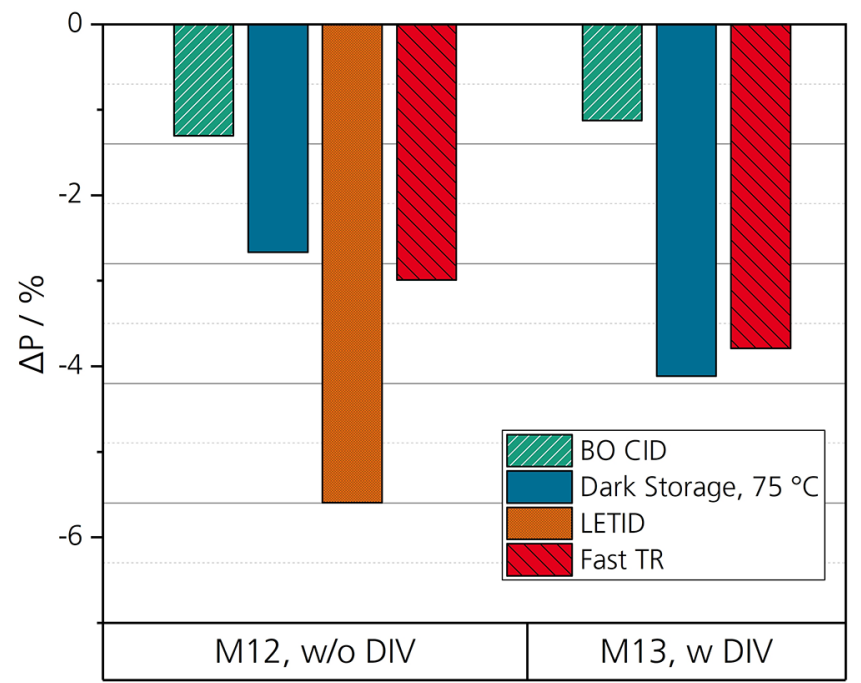

Fig. 11. Relative changes of $P_{M P P}$ at STC of two PV modules, which were tested under Dark Storage at $75^{\circ} \mathrm{C}$ with (M13) and without dark IV monitoring (M12) after BO-CID, Dark Storage, $\mathrm{CID}$ at $75^{\circ} \mathrm{C}$ and Temporary Recovery.

performed at significantly higher temperatures, we consider long-term dark storage at $75^{\circ} \mathrm{C}$ to have a possible influence on subsequent LETID.

Therefore, to distinguish BO LID and LETID after dark storage and CID at $75^{\circ} \mathrm{C}$, a temporary recovery test was performed on module M12 (see Sect. 3.2.7).

\subsubsection{Temporary recovery test}

Temporary recovery experiments have been performed under two different test conditions: In a "slow" recovery test, a current of $I_{S C}-I_{M P P}$ (MPP mode) was injected while the module temperature was controlled at $(25 \pm 3)^{\circ} \mathrm{C}$. As this leads to a similar injection level as Irradiation with one sun in MPP, we expect this condition to cause temporary recovery of the LETID defect $\left(\overrightarrow{B A_{\text {LETID }}}\right)$ at a reaction rate relevant for outdoor exposure at cold, sunny days. In a "fast" recovery test, the current was increased to $I_{S C}$ to complete the recovery process in a reasonable time.

"Fast" temporary recovery at $25^{\circ} \mathrm{C}$ and $I_{S C}$ was used on the modules of the high temperature storage test (M12, M13) to separate LETID from other effects.

The relative changes of $P_{M P P}$ at STC after all experiments, compared to the initial measurement, are shown in Figure 11. The temporary recovery test led to a relative power increase of $2.6 \%$ of module M12, which was tested under high temperature dark storage without dark IV monitoring for $972 \mathrm{~h}$ and was afterwards subjected to $162 \mathrm{~h}$ of LETID testing at $75^{\circ} \mathrm{C}$ in MPP mode. The major losses in the subsequent LETID test interval were therefore most likely caused by LETID. We conclude, that the LETID behavior of this module has possibly been altered by the prior dark storage experiment, which led to significantly stronger LETID than observed for the other modules tested at $75^{\circ} \mathrm{C}$ and MPP mode. 


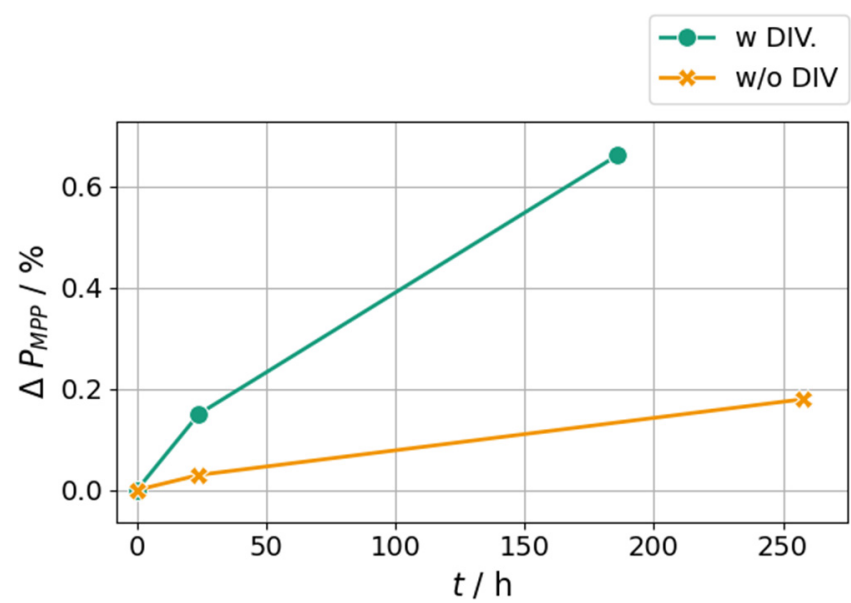

Fig. 12. Relative changes of $P_{M P P}$ at STC of modules M08 (w DIV) and M09 (w/o DIV) during temporary recovery at $25^{\circ} \mathrm{C}$ and $I_{S C}-I_{M P P}$.

In case of module M13, which underwent $1134 \mathrm{~h}$ of dark storage at $75^{\circ} \mathrm{C}$ with in-situ dark IV monitoring, the temporary recovery test led to a relative power increase of only $0.3 \%$. Therefore, the performance loss during the dark storage experiment must have been mainly caused by another effect than LETID. A likely explanation for the power loss is BO LID due to carrier injection during dark IV measurements after prior destabilization of $\mathrm{BO}$ defects during high temperature dark storage (see Sect. 3.2.5).

The modules M08 and M09 were subjected to two intervals of "slow" temporary LETID recovery at $25^{\circ} \mathrm{C}$ and MPP mode after CID at the LETID test conditions $75^{\circ} \mathrm{C}$ and $2 \times\left(I_{S C}-I_{M P P}\right)$ (see Sect. 3.2.4). The objective of this experiment was to investigate temporary recovery at conditions relevant for field exposure.

The relative change of $P_{M P P}$ of the two PV modules is shown in Figure 12. On module M08, dark IV monitoring was performed in-situ during the test. The overall time under dark IV measurements during the experiment was $4.3 \mathrm{~h}$.

While the relative power increase after $258 \mathrm{~h}$ of carrier injection in MPP mode was less than $0.2 \%$ for module M09, almost $0.7 \%$ relative power increase was measured after $186 \mathrm{~h}$ of CID in MPP mode with dark IV monitoring (M08). This is equivalent to an acceleration factor by dark IV monitoring of approximately 5 and indicates a high injection dependency of the process.

The modules M04 and M05, which were subjected to the same recovery experiment after $\mathrm{CID}$ at $75^{\circ} \mathrm{C}$ and $I_{S C}-I_{M P P}$ (see Sect. 3.2.2) gained $0.2 \%$ (with dark IV monitoring, M04) and $\approx 0 \%$ (without dark IV monitoring, M05) during $24 \mathrm{~h}$ of temporary recovery.

After the "slow" recovery experiment, the modules M04, M05, M08 and M09 underwent "fast" LETID recovery at $25^{\circ} \mathrm{C}$ and $I_{S C}$. The intention of using this approach was to recover all LETID and by this identify the magnitude of BO LID and LETID after testing at $75^{\circ} \mathrm{C}, I_{S C}-I_{M P P}$ and at $75^{\circ} \mathrm{C}, 2 \times\left(I_{S C}-I_{M P P}\right)$, respectively. This is important for the further discussion of the difference in the LETID test result (see Sect. 3.2.4).

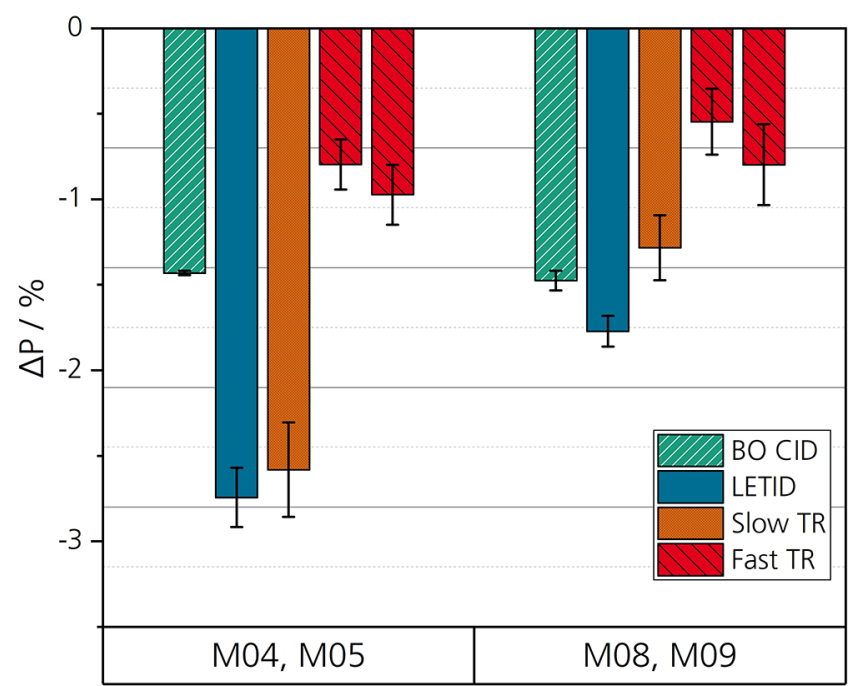

Fig. 13. Relative changes of $P_{M P P}$ at $\mathrm{STC}$ of four PV modules, which were LETID tested at $75^{\circ} \mathrm{C}, I_{S C}-I_{M P P}(\mathrm{M} 04, \mathrm{M} 05)$ and at $75^{\circ} \mathrm{C}, 2 \times\left(I_{S C}-I_{M P P}\right)(\mathrm{M} 08, \mathrm{M} 09)$, respectively, after BO CID, LETID and temporary recovery. Error bars: $\min / \max$ values of two modules.

An overview over the relative $P_{M P P}$ changes of the four modules is given in Figure 13 for all experiments. The values of the relative changes refer to the initial STC measurement before BO CID. After the first $24 \mathrm{~h}$ interval of "fast" temporary recovery, all modules show significant recovery. After a second interval at the same test conditions, a performance decrease between $-0.2 \%$ and $-0.3 \%$ was measured.

As no further recovery was observed after the last test interval, the temporary recovery of LETID has most likely saturated within the first $24 \mathrm{~h}$ of fast recovery. The remaining power loss after the first recovery interval ranges from $-0.7 \%$ to $-0.9 \%$ for the modules M04 and M05, which were recovered after CID at $75{ }^{\circ} \mathrm{C}$ and $I_{S C}-I_{M P P}$. Assuming that the remaining power loss is mainly caused by BO LID, we conclude, that approximately $35 \%$ to $54 \%$ of the BO defects regenerated during 5 weeks of typical LETID test conditions of CID at $75^{\circ} \mathrm{C}$ and $I_{S C}-I_{M P P}$. Similar effects should generally be considered when quantifying LETID after indoor tests since $\mathrm{BO}$ regeneration might reduce the observed performance losses.

In case of the modules recovered after the LETID test at higher current injection with $2 \times\left(I_{S C}-I_{M P P}\right)(\mathrm{M} 08, \mathrm{M} 09)$, the remaining power loss after temporary recovery ranges from $-0.4 \%$ to $-0.7 \%$. The slightly lower remaining performance losses might indicate that a larger share of BO defects regenerated during LETID testing at $75^{\circ} \mathrm{C}$ and $2 \times\left(I_{S C}-I_{M P P}\right)$ compared to the test at $I_{S C}-I_{M P P}$.

However, since the differences in the remaining performance losses after temporary recovery are low, an acceleration of BO LID regeneration of module M08 and M09 due to the increased injection level at $2 \times\left(I_{S C}-I_{M P P}\right)$ can only partly explain the difference in the maximum degradation compared to M04 and M05 during the LETID experiments. 
Stronger differences can be seen in the recovered performance, which can be attributed to LETID. While for the modules M04 and M05 (after LETID testing at $75^{\circ} \mathrm{C}$ and $\left.I_{S C}-I_{M P P}\right) 1.9 \%$ and $2.0 \%$ of $P_{M P P}$ could be recovered, the recovery measured for M08 and M09 (after LETID testing at $75^{\circ} \mathrm{C}$ and $\left.2 \times\left(I_{S C}-I_{M P P}\right)\right)$ amounts $1.1 \%$ and $1.3 \%$.

The largest part of the difference in the LETID test result in Section 3.2.4 can therefore be attributed to a different amount of LETID. One possible explanation could be acceleration of the LETID regeneration due to the higher injection level. However, as mentioned in Section 3.2.4, the influence of the injection level on the magnitude of LETID was significantly lower in other results known from literature.

\section{Outdoor operation}

\subsection{Mono-crystalline PERC PV modules}

The performance changes of the mono-crystalline PV modules in the outdoor experiment (M02, M03) are discussed based on insights from the indoor experiment. The indoor-tested modules of the same type had shown BO LID in the range of $-1.4 \%$ (under CID at $25^{\circ} \mathrm{C}$ and $I_{S C}$, see Sect. 3.2.1) and further performance losses of approximately $-1.3 \%$ under LETID test conditions (CID at $75^{\circ} \mathrm{C}$ in MPP mode, see Sect. 3.2.2). We expect a sensitivity of the outdoor tested modules in the same range. The superimposition of $\mathrm{BO}$ LID and LETID related state transitions and the strong changes in temperature and irradiance over time make the interpretation of the performance changes during the outdoor experiment more complex.

During outdoor exposure, the PV modules were operating at different cell temperatures due to the thermal insulation of one module's rear side. During irradiance of at least $10 \mathrm{~W} / \mathrm{m}^{2}$, the average temperature difference between the insulated and the non-insulated module was $4.8 \mathrm{~K}$ over the so far exposure time. The maximum temperature difference amounted to $20.6 \mathrm{~K}$. More information on the module temperatures during the exposure intervals are given in Appendix B in Supplementary Material.

Other than expected, the not insulated PV module, which was operating at lower cell temperatures, showed stronger performance losses than the insulated PV module during the so far exposure time.

After the first month of outdoor exposure, a relative power loss of $-2.3 \%$ was measured for the non-insulated PV module (Fig. 14). This is surprisingly higher than the performance loss of $-1.7 \%$, which was measured for the thermally insulated PV module. One possible explanation for this observation is a large share of BO LID in the performance loss of the first month.

Since BO regeneration evolves faster than LETID regeneration (see e.g., [7]), the lower performance loss of the insulated PV module might be explained by faster BO regeneration due to the higher temperature level. We conclude that the degradation of $\mathrm{BO}$ defects, which is the fastest process amongst the considered state transitions,
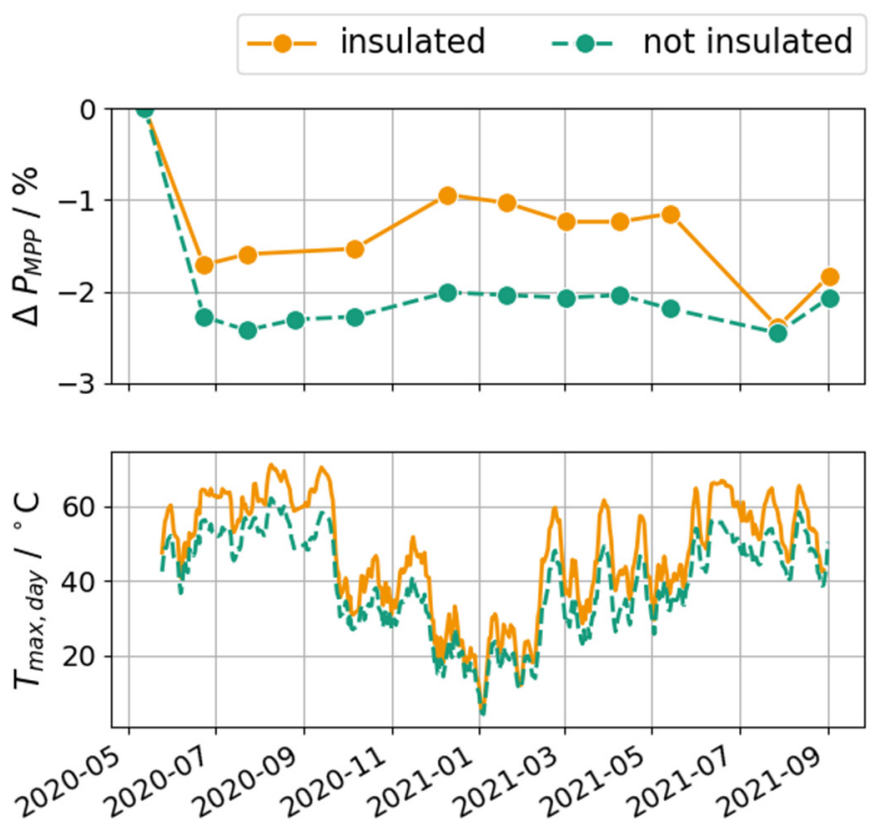

Fig. 14. Performance change over time of two mono-crystalline PERC modules during 15 months of outdoor exposure (top). Weekly average of the daily maximum module temperature (bottom).

was most likely saturated after the first month of operation. During the following summer months, only slight changes are observed with a trend towards performance increase, which is most likely due to further BO regeneration.

During the first cold period, we observed positive performance changes due to temporary LETID recovery on sunny days with low temperatures (see Sect. 3.2.7). From October 9, 2020 to December 12, 2020, positive changes of $0.4 \%$ and $0.2 \%$ were measured. These changes are smaller than the long-term reproducibility of the performance measurement, which is stated to be below $0.5 \%$. The trend of positive performance changes during cold periods is however consistent with observations later described in Section 4.2. The higher power increase of the insulated PV module compared to the ventilated module might indicate a higher share of LETID in the performance loss before the beginning of the cooler period.

The remaining relative performance loss after temporary recovery of the insulated module $(-1.0 \%$, see December 12, 2020) could be either caused completely by BO LID (if all LETID was recovered) or could be a mixture of LID, LETID and possible other effects. Considering the power loss of the modules in the BO CID experiment of $-1.4 \%$, approximately $0.4 \%$ or more performance increase must have evolved due to $\mathrm{BO}$ regeneration during the first summer season.

During the following months, the performance remained almost stable. After the next summer period (May 14, 2021 to September 2, 2021), further performance losses were observed for the insulated PV module. 
After so far 15 months of outdoor exposure, the measured overall performance losses amount $-1.8 \%$ (insulated module) and $-2.2 \%$ (not insulated module). The present power losses of the outdoor modules are thereby close to the overall performance losses measured in the indoor experiment after BO LID Preconditioning and LETID at $75^{\circ} \mathrm{C}$ and $2 \times\left(I_{S C}-I_{M P P}\right)$ (between $-1.8 \%$ and $-2.0 \%$, see Sects. 3.2.1 and 3.2.4) and still below the losses measured after BO LID Preconditioning and LETID at $75^{\circ} \mathrm{C}$ and $I_{S C}-I_{M P P}$ (between $-2.6 \%$ and $-2.9 \%$, see Sect. 3.2.1 and 3.2.2). Therefore, though the future evolution of the modules' performance in the coming exposure time is not known yet, the so far performance changes are in good agreement with results from common indoor test procedures on module level.

\subsection{Multi-crystalline PERC PV modules}

A similar outdoor exposure experiment was also carried out on two poly-crystalline modules, i.e., a different module type than investigated in the indoor and outdoor tests described above.

In a highly accelerated (and therefore less precise) LETID indoor test at $85^{\circ} \mathrm{C}$ and $I_{M P P}$ without prior BO LID preconditioning, two modules of the same type had shown maximum performance losses of $-3.8 \%$ and $-4.2 \%$. Therefore, significant changes due to LETID could be expected in the outdoor test.

The performance changes are discussed with focus on LETID as the expected dominant loss factor and the influence of module temperature.

The thermal insulation of the rear side led to an average temperature difference between the insulated and the not insulated module of $5.9 \mathrm{~K}$ and a maximum temperature difference of $25.4 \mathrm{~K}$ over the so far investigated exposure time. The influence of the rear insulation is highest on warm, sunny days when temperatures above $50^{\circ} \mathrm{C}$, which are most relevant for triggering LETID, can easily be reached. This stresses the importance of the operating temperature for LETID kinetics. More information on the module temperatures during the exposure intervals are given in Appendix B in Supplementary Material.

The first months of operation (April 18, 2019 to October 1, 2019) led to continuous degradation of both PV modules (Fig. 15, top) with the strongest changes of $-2.2 \%$ and $-2.5 \%$ being reached during the first exposure interval. As the reference module, which was covered during storage time, showed $-1.1 \%$ rel. $P_{M P P}$ change over the last two years and two modules of the same type had an average power loss of $-1.6 \%$ during light stabilization according to IEC $61215: 2016-2$, a large share of the degradation during the first weeks of operation might be caused by another LID effect than LETID, such as BO LID.

During the following summer months, the higher operation temperature of the insulated PV module led to faster and stronger performance losses compared to the not insulated PV module. On October 1, 2021, the observed power losses amounted $-4.6 \%$ for the insulated module and $-3.0 \%$ for the module with ventilated rear side.
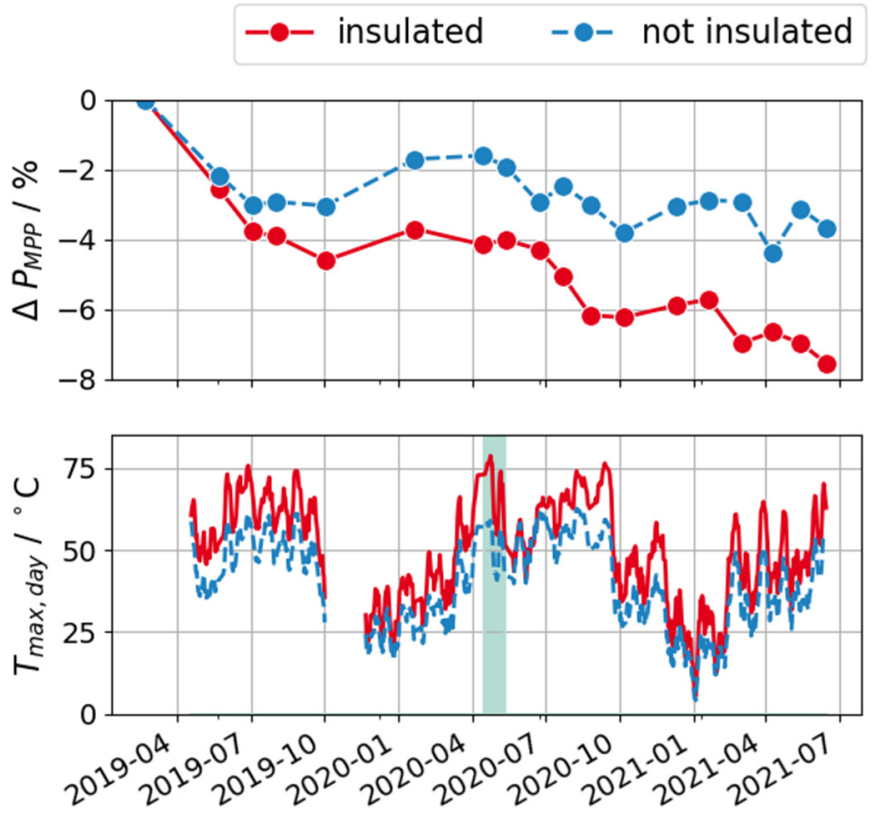

Fig. 15. Performance change over time of two LETID sensitive multi-crystalline PERC modules during two years of outdoor exposure (top). Weekly average of the daily maximum module temperature (bottom). The green colored background highlights a time period with open circuit operation.

After the following winter months, we observed a significant performance increase due to temporary recovery of the LETID defect (see data point January 21, 2020). This is a typical seasonal effect for LETID sensitive PV modules.

Similar effects were also observed in summer 2020 and the following winter.

During the exposure interval between April 17, 2020 and May 12, 2020, the modules were operating in open circuit due to a technical issue. Though unfavorable for PV plants, outdoor exposure under open circuit conditions can happen, e.g., during times of construction, maintenance, or system failure. After operation in $V_{O C}$ at comparably high temperatures, the insulated module showed slight performance increase (0.1\%), which was also visible in the electroluminescence image. The non-insulated module showed a performance decrease of $-0.3 \%$. The performance increase of the insulated module can be either caused by $\mathrm{BO}$ regeneration or LETID regeneration. Both processes are influenced by the injection level of excess charge carriers, which is significantly higher at $V_{O C}$ than in $M P P$.

In case of the insulated PV module, additional losses to LETID contributed to the performance changes in 2021. Between January and March 2021, the insulated module showed a comparably high relative performance loss of $-1.3 \%$ which can be partly attributed to series resistance losses due to finger detachments at cell fractures (see Appendix C in Supplementary Material). Although 
the fractures had already emerged in April 2020, we assume the influence on the module performance before March 2021 to be low.

In June 2021, the measured overall power loss over an outdoor exposure time of two years and two months amounts to $-7.6 \%$ for the insulated PV module (inkl. up to $-1.3 \%$ losses due to finger detachments) and $-3.7 \%$ for the PV module with ventilated rear side. This illustrates the high influence of module temperature on performance losses due to LETID.

\section{Summary and conclusions}

In this work, we investigated the behavior of ten monocrystalline PERC PV modules under indoor experiments and outdoor exposure. Changes in the modules' characteristics were interpreted based on the current knowledge on possible state transitions of the BO defect and LETID.

When interpreting our results, it should be noted, that the magnitude and the kinetics of LETID can vary depending on production processes, e.g., due to thermal history [2,3,25], and therefore cells and modules of the same type can show differences in their LETID behavior. Nevertheless, a comparison based on twelve identically built PV modules tested in five different test sequences and in an outdoor experiment as presented in this work can provide valuable information on the influence of test conditions or operational conditions on the evolution of LID and LETID.

PV modules tested with and without prior BO LID stabilization showed similar cumulative power losses. When only evaluating the losses before and after LETID testing, the difference between prior stabilized and not stabilized modules was approximately $50 \%$. This highlights the importance of a BO pre-stabilization as it was proposed by others [30] and is included in the IEC TS draft [5] for avoiding false fails in the evaluation of LETID tests. A later performed temporary recovery experiment (see Sect. 3.4.7) showed, that also some regeneration of BO LID occurred during the LETID experiments, which could have led to a slight underestimation of LETID if not considered.

An increase of the temperature from $75^{\circ} \mathrm{C}$ to $85^{\circ} \mathrm{C}$ while retaining the current injection at $I_{S C}-I_{M P P}$ resulted in stronger losses. As $85^{\circ} \mathrm{C}$ are rarely reached under outdoor exposure, it is assumed, that testing at $75^{\circ} \mathrm{C}$ is closer to realistic outdoor conditions. It is possible that increasing the temperature above this level has induced further degradation, as it has been assumed in literature [20].

The modules tested at $75^{\circ} \mathrm{C}$ and $2 \times\left(I_{S C}-I_{M P P}\right)$, as prescribed in the IEC TS draft for LETID detection, exhibited lower performance losses than seen at $75^{\circ} \mathrm{C}$ and $1 \times\left(I_{S C}-I_{M P P}\right)$. A subsequent temporary recovery test with CID at $T<30^{\circ} \mathrm{C}$ revealed, that the lower performance loss was primarily due to a lower magnitude of LETID, while faster regeneration of BO LID had a comparably low impact on the difference in the test result. The results suggest that an increased current can lead to an underestimation of power loss due to LETID. However, it should be considered, that the maximum relative performance loss seen under both LETID test conditions after $\mathrm{BO}$ preconditioning was lower than $2 \%$ and that the influence of the injection level on the magnitude of LETID was significantly lower in other studies (e.g., [17,33]).

After a dark storage experiment at $75^{\circ} \mathrm{C}$, performance losses were measured, which can most likely be attributed to the destabilization and annealing of BO defects, as previously observed after damp heat testing [6]. Subsequently to the dark storage experiment, a higher performance loss was observed after $162 \mathrm{~h}$ of CID at $75^{\circ} \mathrm{C}$ and $I_{S C}-I_{M P P}$ compared to the modules tested without prior dark storage. This performance loss could be identified as LETID by applying temporary recovery conditions. If high temperature dark storage causes not only destabilization of BO defects but also leads to higher LETID sensitivity, this process could lead to additional performance losses during the new BO LID stabilization procedure after PID and Damp Heat in IEC61215:2021 [26]. One possible measure could be a subsequent temporary LETID recovery step at $25^{\circ} \mathrm{C}$ and $I_{S C}$ if Gate 2 is failed.

Temporary recovery at $T<30^{\circ} \mathrm{C}$ and carrier injection was highly accelerated by increasing the current from $I_{S C}-$ $I_{M P P}$ to $I_{S C}$. This is consistent with literature, where the process has been shown to be highly dependent on the excess charge carriers [23]. In our experiments, even carrier injection during in-situ dark IV monitoring led to an acceleration at $25^{\circ} \mathrm{C}$ and $I_{S C}-I_{M P P}$.

Under outdoor exposure, the modules were compared with another type of strongly LETID sensitive multicrystalline PERC module. We observed a strong influence of the module's operating temperature on the performance changes. The nature of the impact of the temperature difference was different for the two module types and was most likely influenced by different sensitivities to LETID and BO LID. Since higher temperatures accelerate not only LETID but also the regeneration processes of both LETID and BO LID, high exposure temperatures cause faster and stronger performance losses due to LETID, which fits well with the performance changes observed for the multicrystalline PV modules. In case of the mono-crystalline modules, however, the observation of weaker performance losses of a module with thermal rear side insulation compared with a module with ventilated rear side during the first months of operation could be explained by a faster regeneration of BO LID. If modules exhibit strong BO LID and low LETID sensitivity, higher temperatures could therefore even be advantageous due to the acceleration of $\mathrm{BO}$ regeneration.

All modules showed seasonal effects like performance increase in winter, which can be attributed to temporary recovery of LETID.

After 15 months of outdoor exposure, the power losses of the mono-crystalline PV modules were close to the maximum performance losses observed under indoor LETID tests at $75^{\circ} \mathrm{C}$ and $2 \times\left(I_{S C}-I_{M P P}\right)$. Further exposure time will show, if stronger performance losses will be reached in the third summer period. 
Additional power losses during the coming outdoor exposure time are also possible for both multi-crystalline PV modules, which still showed power losses in the latest performance measurement after a total exposure time of two years and two months. This finding fits well with the timescales of published data for LETID performance losses under field conditions in Germany and Cyprus [34]. Even in warm climates, the evolution of LETID can take years until the maximum power loss is reached. As the regeneration of LETID defects evolves even slower than the degradation, high yield losses due to LETID are likely, especially in warm climates.

\section{Supplementary Material}

Appendix A: In-situ Dark Voltage.

Appendix B: Module temperature during outdoor exposure.

Appendix C: Electroluminescence images of the multicrystalline PV Modules (Outdoor Test).

The Supplementary Material is available at https:// doi.org/10.1051/epjpv/2021009/olm.

The authors would like to thank Sandor Stecklum for his work on the development of the dark IV monitoring setup, Tayyab Naeem for his contributions to the outdoor experiment and all involved colleagues from TestLab PV Modules and CalLab PV Modules, Fraunhofer ISE, who helped with the climate chamber tests and module characterization.

\section{Author contribution statement}

E.F. conceived the design of the experiments in consultation with D.P. and P.G.D.P. and P.G. co-developed the outdoor experiment with E.F.G.M. organized or performed most of the experiments and measurements. E.F. analyzed the data and wrote the manuscript in consultation with P.G. All authors were involved in discussions on the experimental data. D.P. and P.G. provided feedback and corrections to the manuscript.

\section{References}

1. K. Ramspeck, S. Zimmermann, H. Nagel, A. Metz, Y. Gassenbauer, B. Birkmann, A. Seidl, in 27th EUPVSEC, 2012, https://doi.org/10.4229/27thEUPVSEC20122DO.3.4

2. R. Eberle, W. Kwapil, F. Schindler, S.W. Glunz, M.C. Schubert, Energy Procedia 124, 712 (2017)

3. C.E. Chan, D.N.R. Payne, B.J. Hallam, M.D. Abbott, T.H. Fung, A.M. Wenham, B.S. Tjahjono et al., IEEE J. Photovolt. 6, 1473 (2016)

4. D. Bredemeier, D.C. Walter, J. Schmidt, Sol. RRL 2, 1700159 (2018)

5. T. Luka, F. Kersten, M. Pander, M. Koentopp, M. Turek, W. Bergholz, T. Pernau, Towards a test standard of light and elevated temperature-induced degradation, PV Tech Power 23, $53(2020)$
6. F. Kersten, F. Frühauf, R. Lantzsch, M. Schütze, C. Taubitz, F. Fertig, M. Schaper et al., in 15th International Conference on Concentrator Photovoltaic Systems (CPV-15), AIP Conf. Proc. 2147, 090001 (2019)

7. I.L. Repins, F. Kersten, B. Hallam, K. VanSant, M.B. Koentopp, Solar Energy 208, 894 (2020)

8. A. Herguth, G. Schubert, M. Kaes, G. Hahn, in 2006 IEEE 4 th World Conference on Photovoltaic Energy Conversion: Waikoloa, Hawaii, May 7-12, 2006 WCPEC-4, (IEEE, Piscataway NJ, 2006), pp. 940-943

9. H. Hashigami, Y. Itakura, T. Saitoh, J. Appl. Phys. 93, 4240 (2003)

10. P. Hamer, B. Hallam, M. Abbott, S. Wenham, Phys. Stat. Solidi RRL 9, 297 (2015)

11. J. Schmidt, K. Bothe, R. Hezel, in Conference record of the twenty-ninth IEEE photovoltaic specialists conference: 2002 (IEEE, 2002), pp. 178-181

12. S.W. Glunz, E. Schaffer, S. Rein, K. Bothe, J. Schmidt, in 3rd World Conference on Photovoltaic Energy Conversion, 2003 (2003)

13. S. Wilking, C. Beckh, S. Ebert, A. Herguth, G. Hahn, Solar Energy Mater. Solar Cells 131, 2 (2014)

14. V. Steckenreiter, D.C. Walter, J. Schmidt, AIP Adv. 7, 35305 (2017)

15. B. Hallam, M. Abbott, N. Nampalli, P. Hamer, S. Wenham, J. Appl. Phys. 119, 65701 (2016)

16. S. Wilking, M. Forster, A. Herguth, G. Hahn, Solar Energy Mater. Solar Cells 142, 87 (2015)

17. F. Kersten, P. Engelhart, H.-C. Ploigt, A. Stekolnikov, T. Lindner, F. Stenzel, M. Bartzsch et al., Solar Energy Mater. Solar Cells 142, 83 (2015)

18. D. Bredemeier, D. Walter, J. Schmidt, Solar Energy Mater. Solar Cells 173, 2 (2017)

19. W. Kwapil, T. Niewelt, M.C. Schubert, Solar Energy Mater. Solar Cells 173, 80-84 (2017)

20. T. Luka, M. Turek, C. Hagendorf, Solar Energy Mater. Solar Cells 187, 194 (2018)

21. C. Vargas, G. Coletti, C. Chan, D. Payne, Z. Hameiri, Solar Energy Mater. Solar Cells 189, 166 (2019)

22. F. Kersten, P. Engelhart, H.-C. Ploigt, F. Stenzel, K. Petter, T. Lindner, A. Szpeth, M. Bartzsch, A. Stekolnikov, M. Scherff, J. Heitmann, J.W. Müller, in 31st EUPVSEC 2015, https://doi.org/10.4229/EUPVSEC20152015-5CO.16.5

23. W. Kwapil, J. Schon, T. Niewelt, M.C. Schubert, IEEE J. Photovolt. 10, 1591 (2020)

24. T.H.Fung, C.E. Chan, B.J. Hallam, D.N. Payne, M.D. Abbott, S.R. Wenham, Energy Procedia 124, 726 (2017)

25. C. Chan, T.H. Fung, M. Abbott, D. Payne, A. Wenham, B. Hallam, R. Chen et al., Sol. RRL 1, 1600028 (2017)

26. International Electrotechnical Commission, IEC61215$2: 2021(2021)$

27. E. Fokuhl, T. Naeem, A. Schmid, P. Gebhardt, T. Geipel, D. Philipp, in 36th EUPVSEC, 2019 https://doi.org/10.4229/ EUPVSEC20192019-4BO.12.2

28. J. Karas, I. Repins, M.B. Koentopp, F. Kersten, J.-N. Jaubert, C. Monokroussos, L. Jakisch et al., First Results from the International Round Robin Study on Light- and Elevated Temperature-Induced Degradation (LETID) (Poster at 2021 PV Reliability Workshop, 02/22-26)

29. TÜV. Rheinland, TÜV 2 PfG 2689/04.19: Light and Elevated Temperature Induced Degradation (LeTID) Test for c-Si Photovoltaic (PV) Modules: Detection (2019) 
30. M.B. Koentopp, F. Kersten, E. Herzog, Towards an IEC LETID Test Standard: Procedures, Kinetics, and Separation of B-O Degradation from LETID (Poster at 2020 PV Reliability Workshop)

31. E. Fokuhl, T. Naeem, P. Gebhardt, D. Philipp, Light and elevated Temperature Induced Degradation (LeTID) - Ein Vergleich von Prüfmethoden (Poster at 35th PV Symposium Bad Staffelstein, 2020)
32. M. Wolf, G.T. Noel, R.J. Stirn, IEEE Trans. Electr. Dev. 24, 419 (1977)

33. G.M. Wyller, M. Wiig, I. Due-Sorensen, R. Sondena, IEEE J. Photovolt. 11, 878 (2021)

34. F. Kersten, F. Fertig, K. Petter, B. Klöter, E. Herzog, M.B. Strobel, J. Heitmann et al., Energy Procedia 124, $540(2017)$

Cite this article as: Esther Fokuhl, Daniel Philipp, Georg Mülhöfer, Paul Gebhardt, LID and LETID evolution of PV modules during outdoor operation and indoor tests, EPJ Photovoltaics 12, 9 (2021) 